Military Technical College Kobry El-Kobbah, Cairo, Egypt.

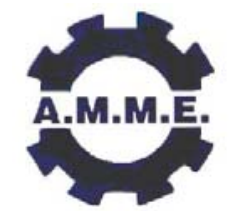

$13^{\text {th }}$ International Conference on Applied Mechanics and Mechanical Engineering.

\title{
USING MULTIVARIATE LATENT MODELS TO MONITOR A PRINTING MACHINE AND PREDICT MACHINE FAILURE
}

\author{
SAID E.M., HUSSEIN W.M., SALEM A.M., MOHAMED K.I.
}

\begin{abstract}
Condition-Based Monitoring involves continuous collection and interpretation of data relating to the operating conditions of critical components of the machine. To ensure high printing quality while maximizing productivity, an on-line process monitoring system is required to take the place of an expert's judgment. This paper outlines the use of a statistical multivariate technique called Principal Component Analysis (PCA), as a health monitoring technique, and applies it to monitor a GTO type of printing machine. This approach is used to integrate vibration data taken at different positions and directions on the printing machine. Experiments were conducted on the machine for different operating speeds under two conditions, new and worn drive belt. The results showed that the proposed technique can be used for printing machine monitoring and can successfully differentiate between new process conditions.
\end{abstract}

\section{KEY WORDS}

Printing monitoring, multivariate analysis, Principal Component Analysis, Vibration monitoring. 


\section{INTRODUCTION}

Printing machines are complicated in design and construction and they consist of very expensive parts. They include many rollers and cylinders driven by an exposed gear train system and they also have various rotating parts like, gears, shafts, bearings, drums and other rotating elements. These rotating parts generate mechanical forces during normal operation. As the mechanical condition of the machine is changed, due to variation of its technical state, changes in operating environments, load variations, etc., a variation in these forces will be occurred. Vibration analysis is most commonly used for machine condition monitoring. Vibration in machines is caused by cyclic excitation forces arising from imbalances, wear, or failure of parts..... etc. Understanding machinery dynamics and vibration and how forces create unique vibration frequency components has been the topic of several research studies in the past [1-4]. In this work, a statistical multivariate technique called Principal Component Analysis (PCA) is applied to a GTO type of printing machine as a health monitoring technique in order to save effort and money spent through traditional techniques used for machine monitoring. The driving system of the printing machine is taken into consideration as the most effective system on the machine vibration signature.

The driving system of the printing machine under investigation, as shown in figure 1 , consists of an electric motor with driving pulley $\mathrm{P} 1$, main driving belt $\mathrm{B} 1$ which connects pulley $\mathrm{P} 1$ with pulley $\mathrm{P} 2$. The pulley $\mathrm{P} 3$ is fixed on the same shaft of pulley $\mathrm{P} 2$ and it is connected with pulley $\mathrm{P} 4$ through secondary driving belt B2. A pinion gear $\mathrm{G} 1$ transmits the torque to the machine drums through gears $\mathrm{G} 2, \mathrm{G} 3, \mathrm{G} 4$, and $\mathrm{G} 5$. The plate cylinder is connected to gear G5; the blanket cylinder is connected to gear G4, while the impression cylinder is connected to gear G3.

Most monitoring techniques presently available describe the variation of one or a limited number of machine components using a heuristic approach which gives limited information about machine condition. Among these techniques, artificial neural network ANNs and its combination with other methods have been the most predominant to date $[5,6]$. ANNs techniques are characterized as black box approaches which model the relation between different variables to a desired response without giving any information about what happens inside the process.

Also some of these approaches are susceptible to missing data due to sensor reliability issues, and some applications use many charts to monitor individual process. In addition, issues associated with collinearity and dimensionality need to still be specifically addressed in these techniques. Looking for a generic approach to solve many of these limitations can be addressed through the use of multivariate statistical analysis [7]. Multivariate statistical process control (MSPC) [8], takes a different approach as compared to the other methods mentioned previously. The most fundamental difference with this approach is that the model is based on non-causal empirical correlations extracted from normal plant operating data when only common cause variation exists. The simplicity of this approach is that there is no need for a fundamental model of the system and only data from normal production needs to be used, which is generally available in some form at most factories. Among the approaches used in multivariate analysis are: a projection method which called principal component analysis (PCA) $[9,10]$ and projection to latent structure (PLS) [11]. Many applications of these two techniques have been successfully applied in other fields of process monitoring $[5,12-17]$ ranging from batch to continuous processes. 


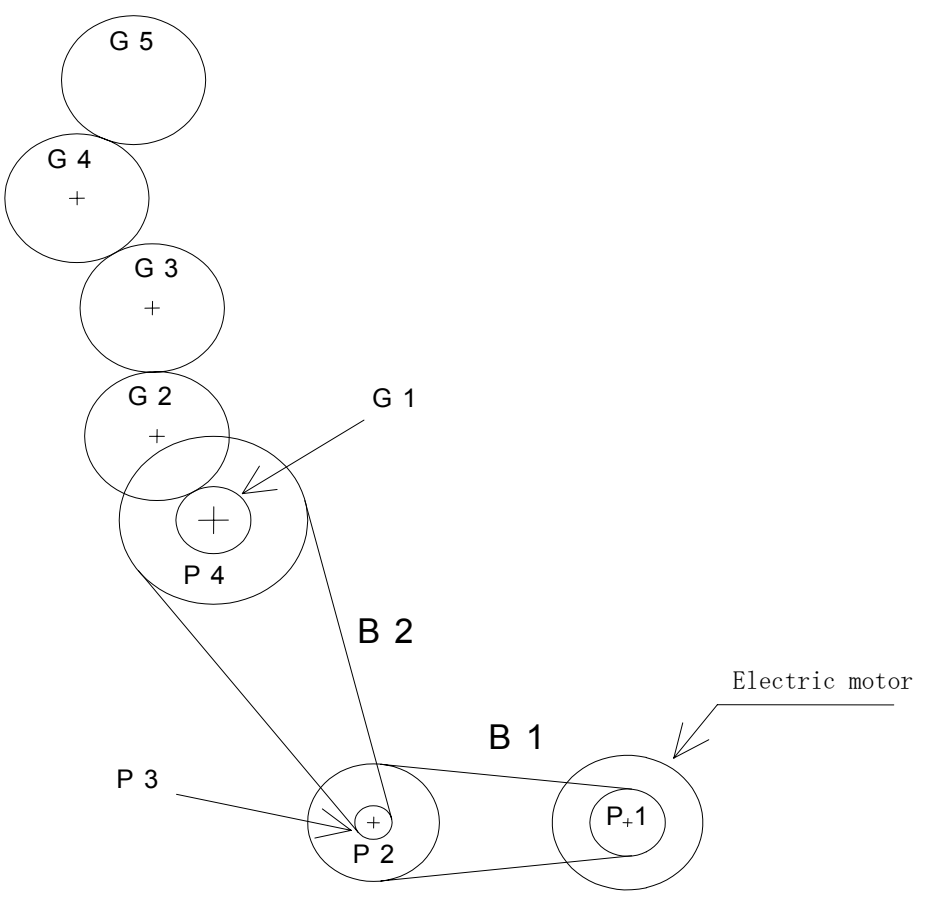

Figure 1: Scheme drawing for GTO driving system

This paper outlines the use of multivariate projection approach to develop a framework to monitor the printing machine under study. By integrating different data collected from different accelerometers fixed at different machine positions and directions under different operating speeds and conditions. Models based on PCA can be developed into a single user interface at which, machine performance can be monitored using the latent space of these models.

\section{MULTIVARIATE PCA BASIC THEORY}

Principal component analysis decomposes the variance and covariance structure of a data matrix by defining linear combinations of the columns in the original matrix. Moreover, PCA extracts information from data sets by computing a smaller data set and other summary information that adequately captures most of the underlying features from the larger data set. The point that needs to be stressed is that the data can be reduced to a size which is more manageable but contains the features that are often of interest. A mathematical description of PCA is required to understand this approach. Let the original data set be represented by the matrix, $\mathrm{X}$, with $\mathrm{N}$ rows and $\mathrm{K}$ columns. PCA extracts a score matrix, $T$, and a loading matrix, $P$, from $X$. These matrices have the following dimensions:

$$
\mathrm{X}: \mathrm{N} \times \mathrm{K} \quad \mathrm{T}: \mathrm{N} \times \mathrm{A} \quad \mathrm{P}: \mathrm{K} \times \mathrm{A}
$$

The matrices $T$ and $P$ cannot have more columns than the original data set, as $A \leq K$; the reduction in the data size is achieved by choosing $A$ to be smaller than $\mathrm{K}$.

We call the first column of $T$ and $P$ by their shorter forms, $t_{1}$ and $p_{1}$ respectively. The lower case letters indicate that these are vectors while the upper case letters indicate matrices. 
Extracting only one principal component (that is, a single score vector and loading vector) gives: $\quad X=t_{1} p_{1}^{T}+E 1$ and extracting a second principal component:

$$
X=t_{1} p_{1}^{T}+t_{2} p_{2}^{T}+E 2
$$

We continue in this manner until we extract principal components and then group these score and loading vectors to form matrices $\mathrm{T}$ and $\mathrm{P}$ :

$$
T=\left[t_{1} t_{2} \ldots t_{A}\right] \text { and } P=\left[p_{1} p_{2} \ldots p_{A}\right]
$$

More compactly then: $\quad X=T P^{\top}+E_{A}$. If we compute as many principal components as there are columns in the data set $X$, then we can directly write that $X=T P^{\top}$, but in this case the amount of data that we have to deal with in $T$ and $P$ is the same as what we started with. The only difference is that it is in a more useful form. Specifically, the principal components are orthogonal to each other so that information captured in principal component 1 is not repeated in any subsequent components. We can also monitor statistics on the residual matrices, $\mathrm{E}_{\mathrm{i}}$, to ensure that all significant information of interest has been extracted by the principal components. What remains now is for us to actually compute the columns of $T$ and $P$. There exists a very fast algorithm for computing these principal components when given a data set that has many more rows than columns, $\mathrm{N} \gg \mathrm{K}$. Consequently we will only mention and deal with this method. The eigenvectors of the real symmetric matrix $X^{\top} X$ give us exactly the loading matrix $P$. This is the loading matrix obtained by extracting all principal components. Once we have $\mathrm{P}$ we use the last line of the following relationship to compute $\mathrm{T}$ :

$$
\mathrm{TP}^{\mathrm{T}}=\mathrm{X} \quad \mathrm{TP}^{\mathrm{T}} \mathrm{P}=\mathrm{XP} \quad \mathrm{T}=\mathrm{XP}
$$

This works because $X^{\top} X$ is a real symmetric matrix and the eigenvectors from such a matrix have the property that $P^{\top} P=I$, with I being an identity matrix. The speed of this algorithm stems from the fact that the eigenvectors are computed on a very small matrix of size $\mathrm{K} \times \mathrm{K}$ and that we do not have to use the original $\mathrm{N} \times \mathrm{K}$ matrix. Most eigenvector calculation routines also provide the eigenvalues. The largest eigenvalue is associated with the first principal component, the second largest eigenvalue with the second component, and so on. A more in-depth discussion, which also highlights some geometric concepts of PCA, can be found in [9]. The algorithm used to calculate the PCA is the NIPALS (Nonlinear Iterative Partial Least Squares) algorithm [10].

\section{EXPERIMENTAL SET-UP}

Measurements were performed on the GTO HEIDELBERG one color (one unit) printing machine. It is to be driven by a belt pulley drive system using an AC motor with $1.5 \mathrm{HP}$ and constant rotating speed of $1440 \mathrm{rpm}$.

The experimental setup is used to fulfill the objective of monitoring the machine by carrying out vibration measurements at different positions and different operating speeds. A mechanical tachometer, engaged with the axis of the driven pulley P4, is used to measure machine speed. After determining the speed of the driven pulley P4, it can be easy to calculate the plate and blanket cylinder speed. The vibration was 
measured using a piezoelectric accelerometer; Figure 2 shows a photograph for the experiment setup.

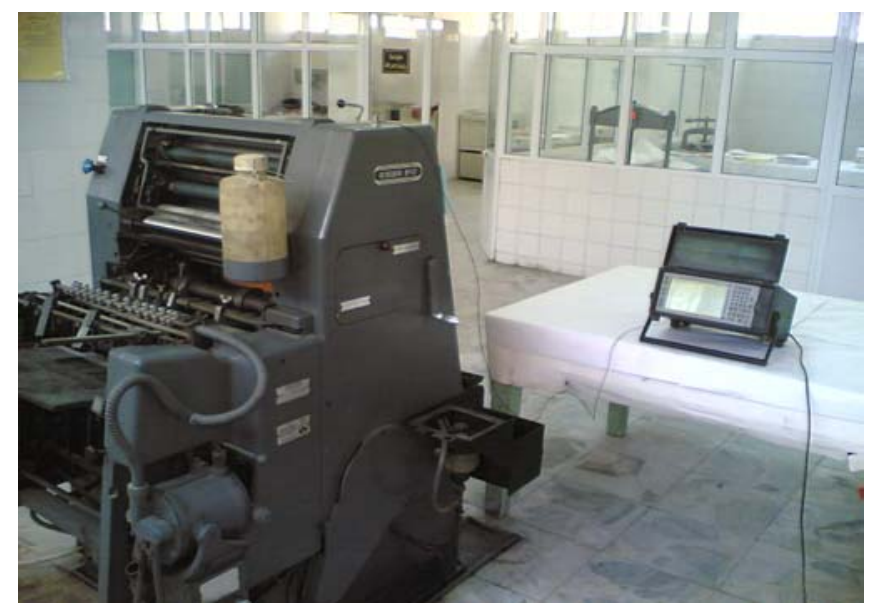

Figure 2: Experimental test setup

Measurements were performed on the printing machine at different positions; these positions were chosen in such a way that optimum results have been obtained and hence ensure accurate signal analysis. Six different locations are chosen for mounting the accelerometer taking into consideration the drive side (Pos. 1,3,5,6) and the control side (Pos. 2,4) as shown in figure 3 . These locations provide good information about the machine vibration. Measurements were performed at each position in three basic directions $\mathrm{x}, \mathrm{y}$ (radial direction) and $\mathrm{z}$ (axial direction) and at different operating speeds (low, medium and high), for a good and defected machine condition.

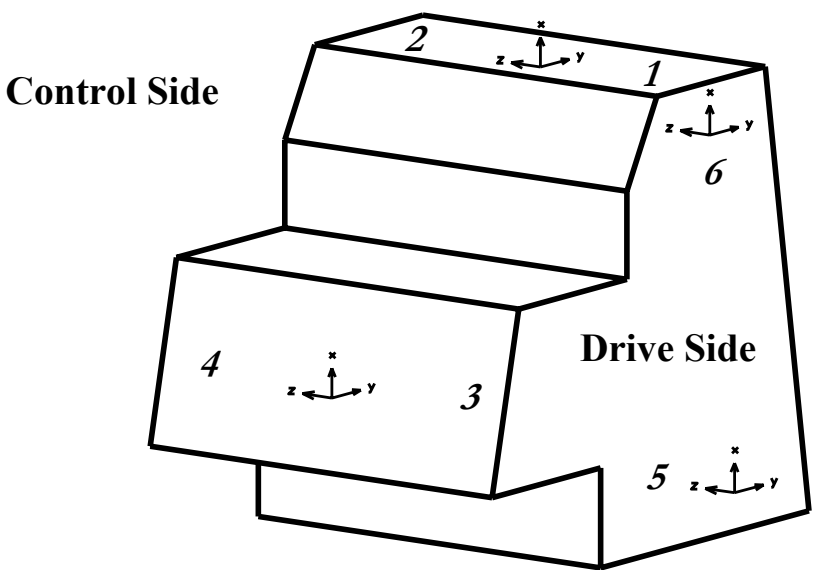

Figure 3: Predefined measurement positions

\section{MODEL BUILDING AND CONFIGURATION}

The multivariate PCA model is a linear model given in matrix form by: $X=T P^{\top}+E_{A}$. However, there exist non-linear PCA versions even by augmenting the original matrix with the non-linear factors or by building a non-linear relation between score factors 
[10]. To build the model using process variables, let $X$ include variables containing the information in the sensory data.

There are different approaches to deal with the available data depending on their type. The simplest way is to use only normal data based on common cause variation and build the model, then use the model to detect any shift or fault that will violate the correlation structure established between the variables during the process. Then by examining the contribution plots we can isolate the variables known as X-predictors that are most responsible for this shift.

\section{RESULTS AND ANALYSIS}

In this section, experimental data are analyzed using PCA. This approach is intended to analyze sensory data from the six positions to construct a multi-sensor monitoring system. This system is tested against belt wear and different machine speeds. First, all data were mean centered and scaled to unit variance. Second, control limits in the latent space were established using F-distribution based on reference distribution provided by the dataset [18]. Also, $t_{1}$ and $t_{2}$ are the first two principal components that capture most of the variance in the $X$ matrix. Figure 4 illustrates how the score plot is built for a simple case 3-variables and 2-scores. After determining the direction of maximum variation by iterative steps and get the second orthogonal direction by the same way after subtracting the first component, we rotate the new plane determined by the new score variables $t_{1}$ and $t_{2}$ and then monitor the movement of the process variables in the reduced dimensional space during printing.

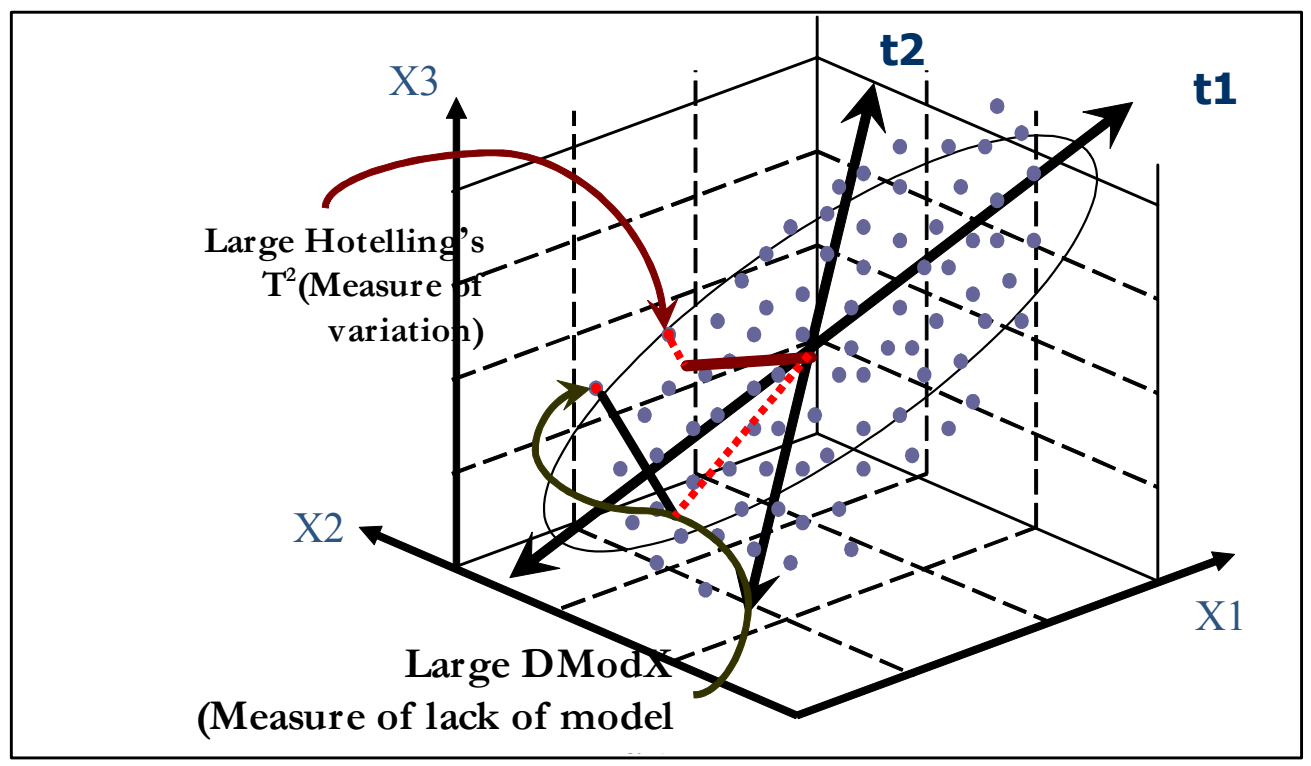

Figure 4: Establishing score plot for three variables

A general scheme of the simulated monitoring system is shown in figure 5 . In this work, experimental data are analyzed as multiple sensory data (six positions). 


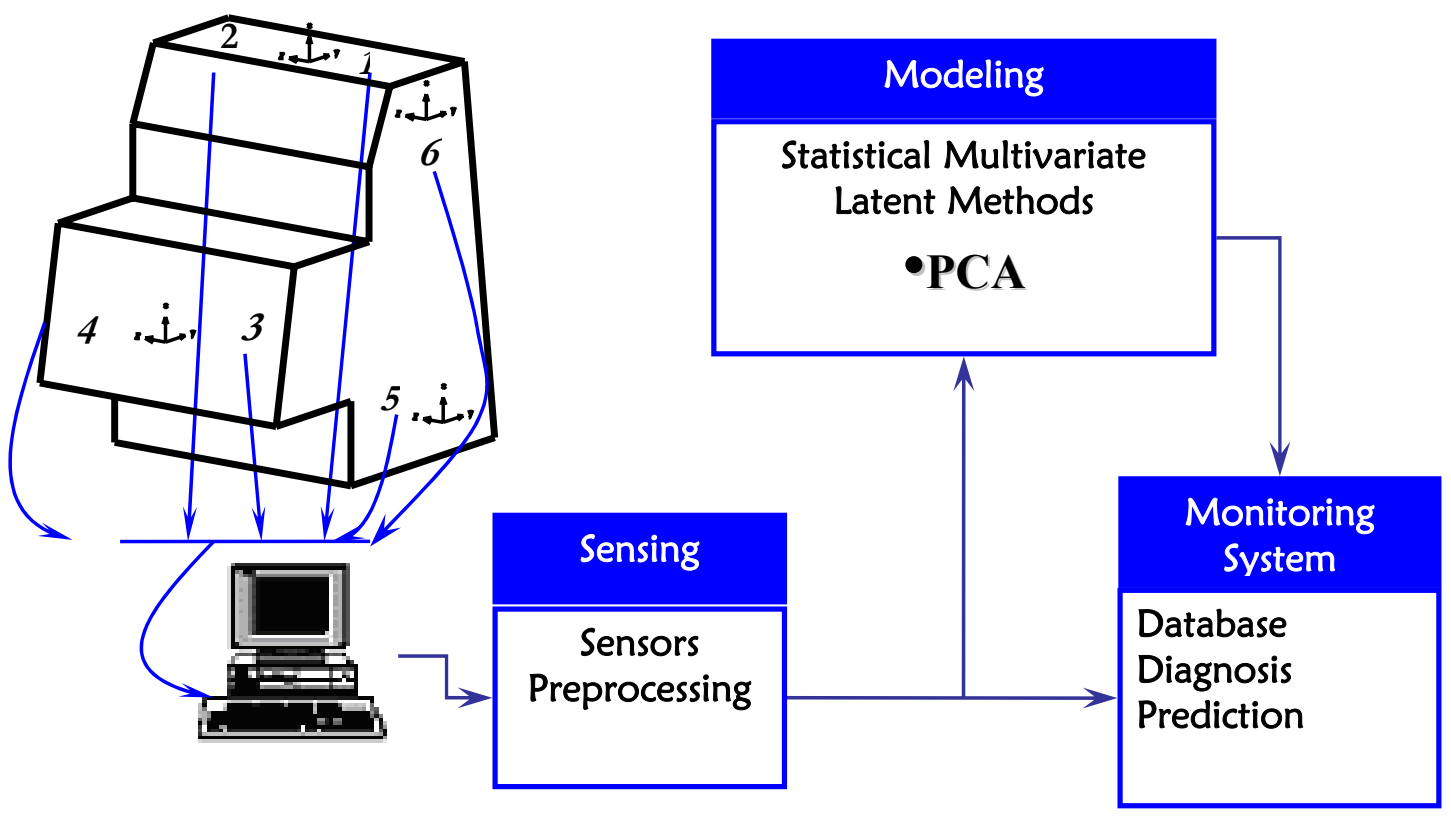

Figure 5: General scheme of proposed monitoring system

A total of 18 variables were used to generate the input matrix " $X$ " of these variables, six represent the six different positions from time domain records (length 1024) of the vibration signals. At each position, data were collected in three directions $\mathrm{x}, \mathrm{y}$ and $\mathrm{z}$. Available groups of data are new belt and worn belt at different machine speeds (low, medium and high speeds). All of the models were built using SIMCA-P code developed by Umetrics [7] based on PCA. The PCA model is based on building classes or clusters using existing information inside the data (unsupervised learning). The model is established using a set of experimental runs called the training set that represents the normal operation of the machine. Finally, regression is made on the matrix $X$ based on NIPALS algorithm.

First, it is decided to start with all the available groups of data which are from new and worn belt data sets for the different machine speeds as shown in figure 6 .

\begin{tabular}{|c|c|c|c|c|c|c|c|c|c|c|c|c|c|c|c|c|c|c|c|}
\hline & & $\mathrm{x} 1$ & $\mathrm{y} 1$ & $\mathrm{z} 1$ & $\mathrm{x} 2$ & $\mathrm{y} 2$ & $\mathrm{z} 2$ & $\mathrm{x} 3$ & $\mathrm{y} 3$ & $\mathrm{z} 3$ & $\mathrm{x} 4$ & $\mathrm{y} 4$ & $\mathrm{z} 4$ & $\mathrm{x} 5$ & $\mathrm{y} 5$ & $\mathrm{z} 5$ & $\mathrm{x} 6$ & $\mathrm{y} 6$ & $\mathrm{z6}$ \\
\hline \multirow{5}{*}{ New } & Low & & & & & & & & & & & & & & & & & & \\
\hline & Med & & & & & & & & & & & & & & & & & & \\
\hline & High & & & & & & & & & & & & & & & & & \\
\hline \multirow{3}{*}{ Worn } & Low & & & & & & & & & & & & & & & & & & \\
\hline
\end{tabular}

Figure 6: X- Matrix for PCA model

The PCA model was built using all the dataset by cross-validation, now obtaining a two component model, with: R2X (cum): 0.55, Q2 (cum) : 0.383. It is seen that the model factors R2X and Q2cum, are very small, but it is still better to first get a general look at the whole dataset without removing any variable or observation. Figure 7 illustrates a scatter plot of the two score vectors ( $\mathrm{t} 1$ and $\mathrm{t} 2$ ) of the PCA model using a set of 6144 
observations. According to the different clusters in figure 7, it can be seen that the discrimination is between six groups of data. Also, a clear discrimination exists between the two different conditions (normal and worn belt) under different speeds (low, high and intermediate). This clustering is shown by a straight line separating the different conditions (normal and worn belt operation). More analysis is made by examining the loading plot which shows the relation between the different variables. Figure 8 shows the loading plot clarifying the relation between the 18 variables. In addition, the score and loading plots are superimposed; this means that variables lying in each quarter of the loading plot are contributing to the changes between the observations in the score plot. So, by investigating the loading plot (figure 8), one can detect directly which variables are responsible or more affecting a specified group of data in the score plot. However, the model doesn't include the effect of speed so it is hard to set a relation between different clusters and the 18 variables. From these two figures, one can notice, for example, that variables Ax4, Ax5, Ay1 and Ay6 in the left corner of the loading plot contribute to the left swarm of data in the score plot. In other words, these variables represent the different sensory positions and directions that give the highest level of vibration during monitoring these set of data swarm.

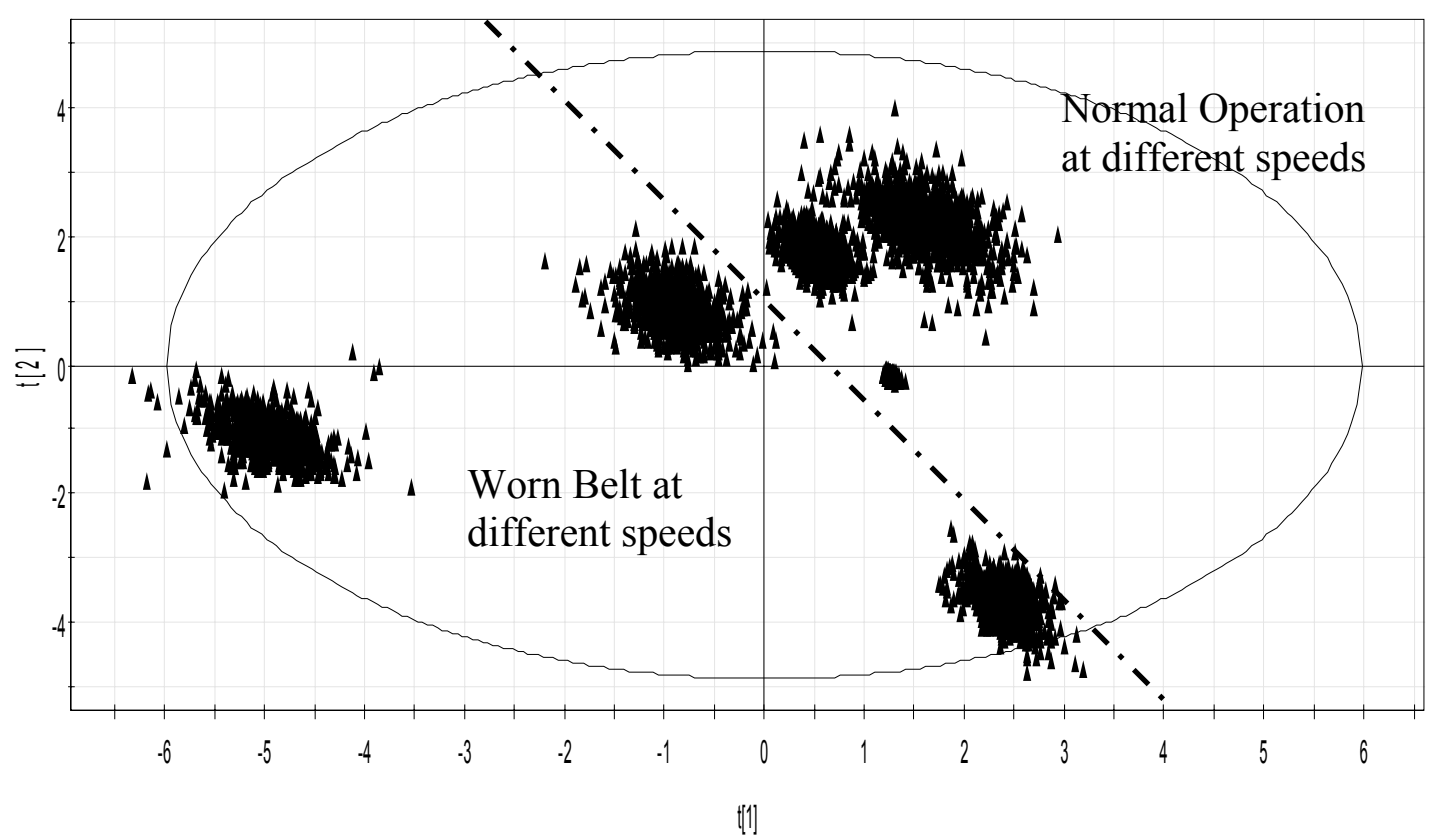

Figure 7: Score plot of PCA model for new and worn belt data (different speeds)

Further analysis of the score plot in a line style figure 9 reveals the main reason for the six clusters. One can notice the six steps of the signal line in the direction of the first score t1. These steps represent the different machine speeds (Low, medium and high speeds) in both conditions new and worn belt.

In this work, the main target is to build a monitoring system to the printing machine. Also, the PCA approach is based on using available database that give an acceptable level of process quality to build the monitoring system and then the model can detect any fault or shift from normal operation. Therefore, the same setting is repeated again with the use of data of the new belt that gives an acceptable printing quality. A total of 18 variables were used in this analysis with the first 3072 observations representing the 


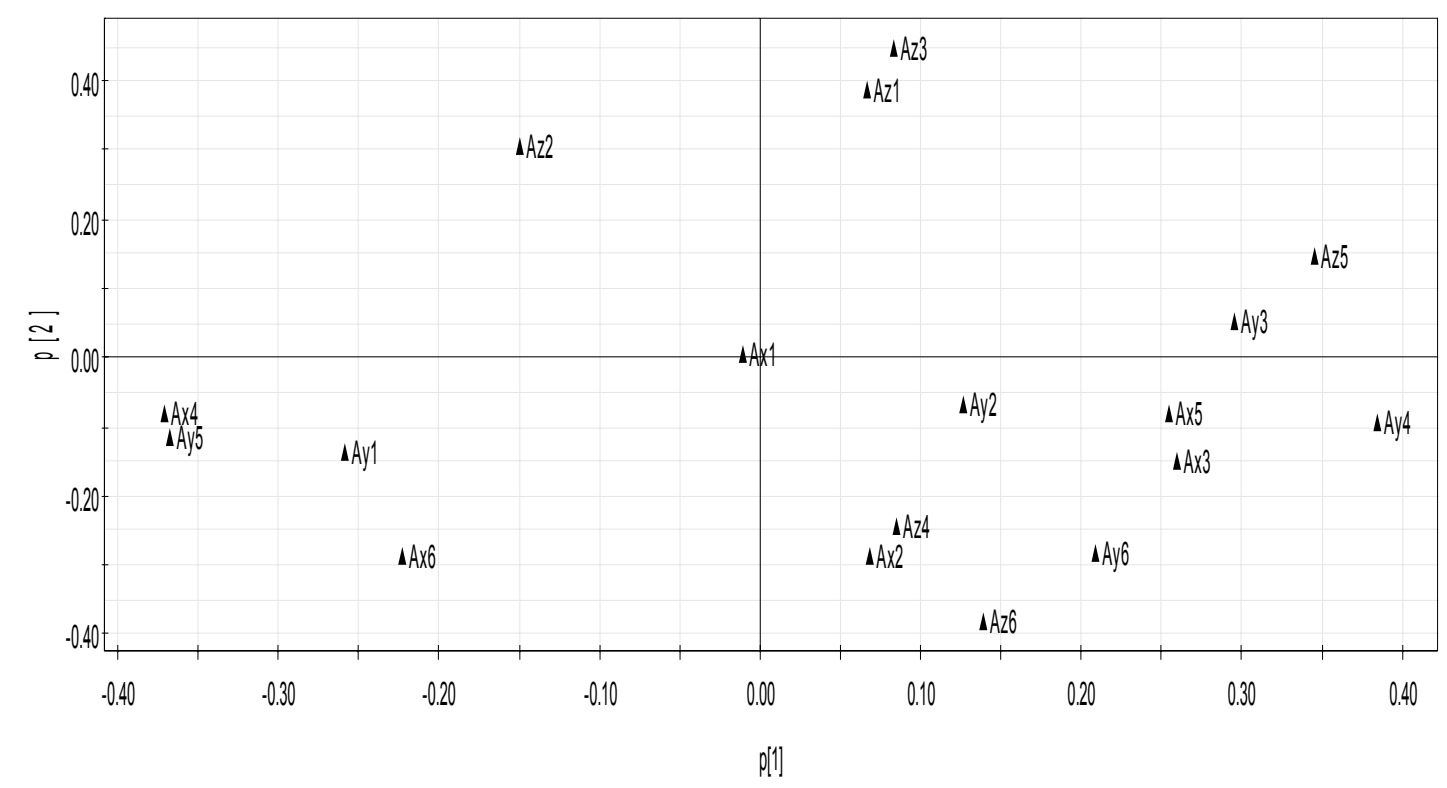

Figure 8: Loading plot of PCA model for and worn belt data (different speeds)

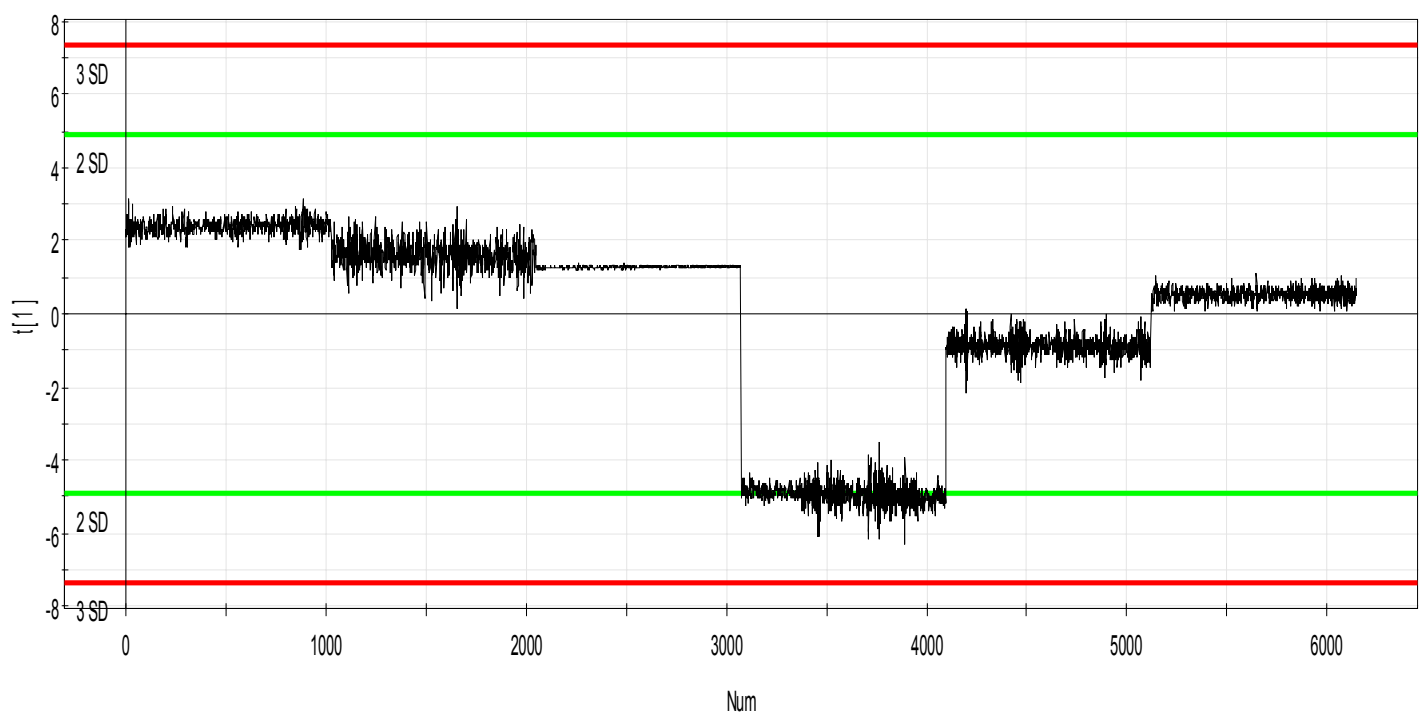

Figure 9: Score line-plot of PCA model for new and worn belt data (different speeds)

normal operation with the new belt. A new model was fitted by cross-validation to get a two component model, with: R2X(cum): 0.724, Q2(cum): 0.664. A clear improvement has been made for the percentage of explanation of the X-variables R2Xcum, together with the prediction ability as shown by Q2cum. Figure 10 illustrates a scatter plot of the two score vectors ( $\mathrm{t} 1$ and $\mathrm{t} 2$ ) of the PCA model using the new belt data set. According to the manually highlighted point clusters in figure 10 (done only for visual purposes), it can be seen the discrimination between three main clusters representing data at different machine speeds. This result is confirmed from figure 11 which illustrates the score line plot. One can notice the three steps in the data line representing different machine speeds low, medium and high respectively. 


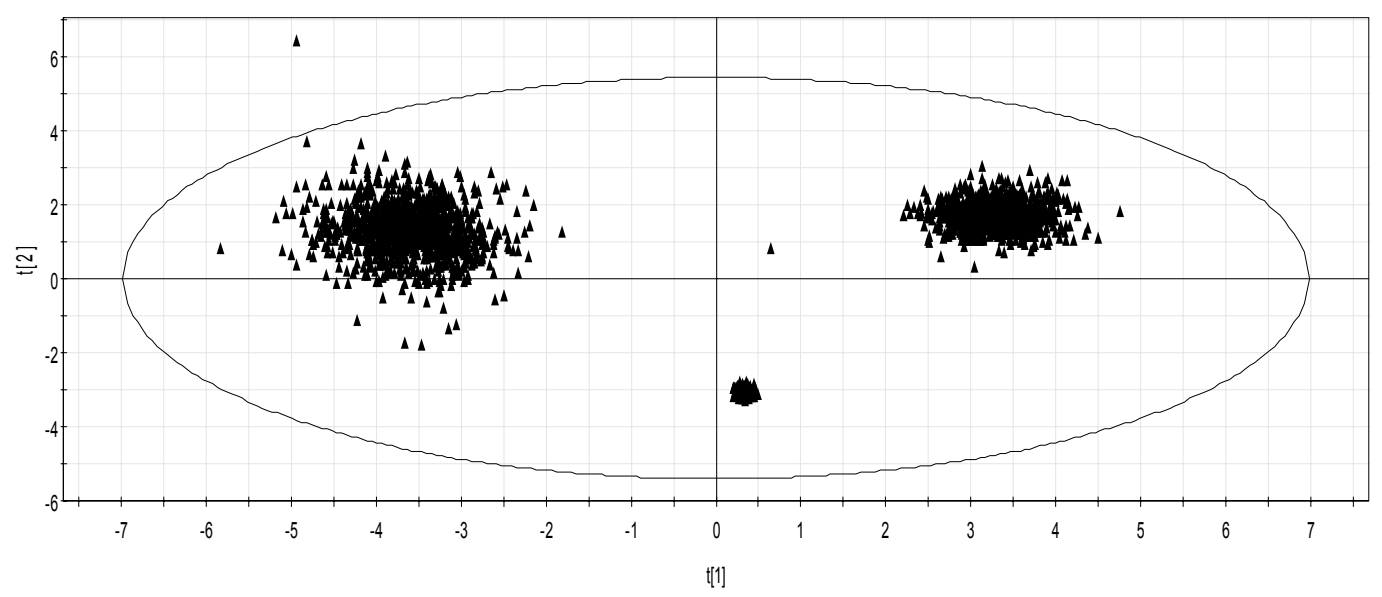

Figure 10: Score plot of PCA model for new belt data (different speeds)

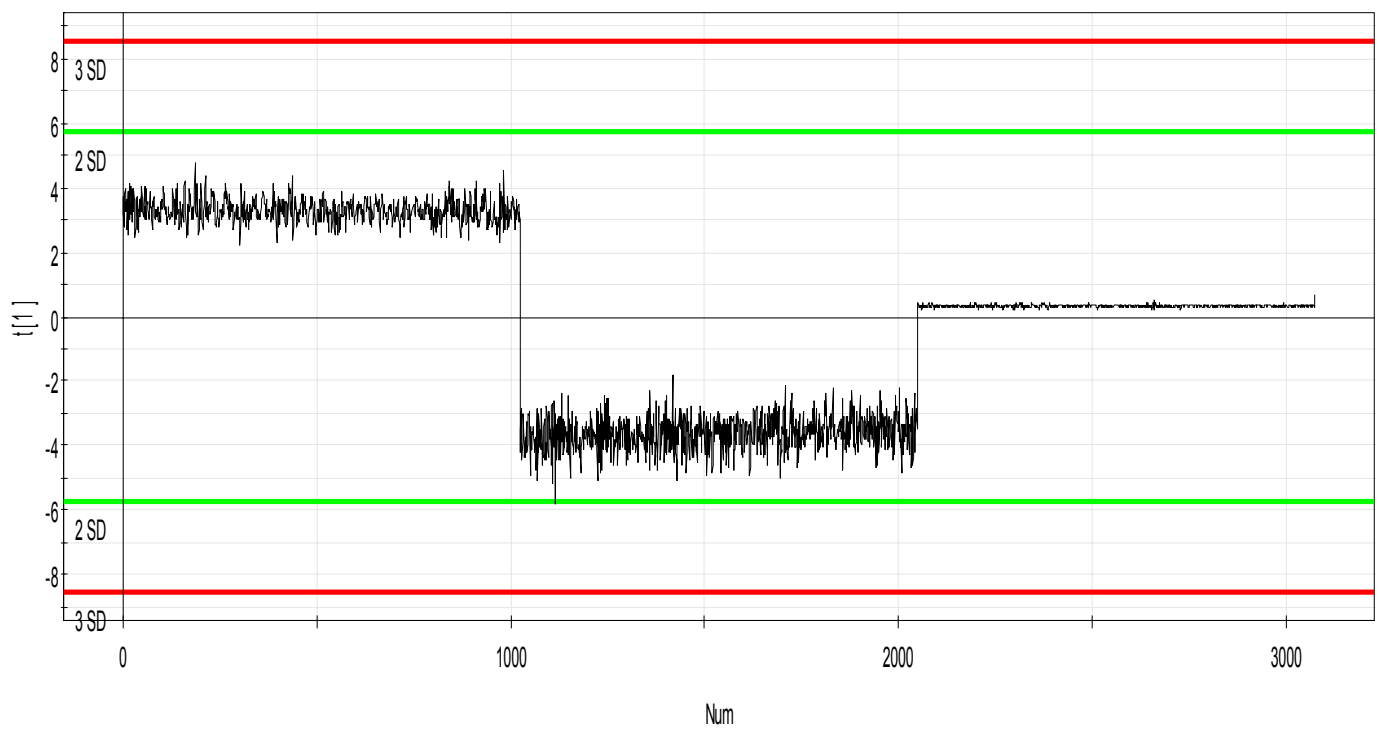

Figure 11: Score line-plot of PCA model for new belt data (different speeds)

More investigation is made using the loading plot, figure 12. In this plot together with column plot (figure 13), one can detect directly how different measurement positions and directions affect the vibration levels at different machine speeds. Also, figure 13 provides how well the model can explain and predict these different variables. Consequently, by knowing this information, one can choose between the different positions and directions of the sensory measurements that most dominate. Dominant factors are the factors that have the longest distance from the origin. For example, Az1 and $A \times 6$ are of the dominant variables.

By examining figure 13, one has different opportunities to choose between different factors that can build a good monitoring system with the highest percentage. However, a rule of thumb in constructing a monitoring system is to minimize the number of sensory positions as much as possible. In addition, one would choose different positions that cover the whole system. In this study, we chose Az1, Ax2 and Ax6 as a simulated sensory system to monitor the printing machine. 


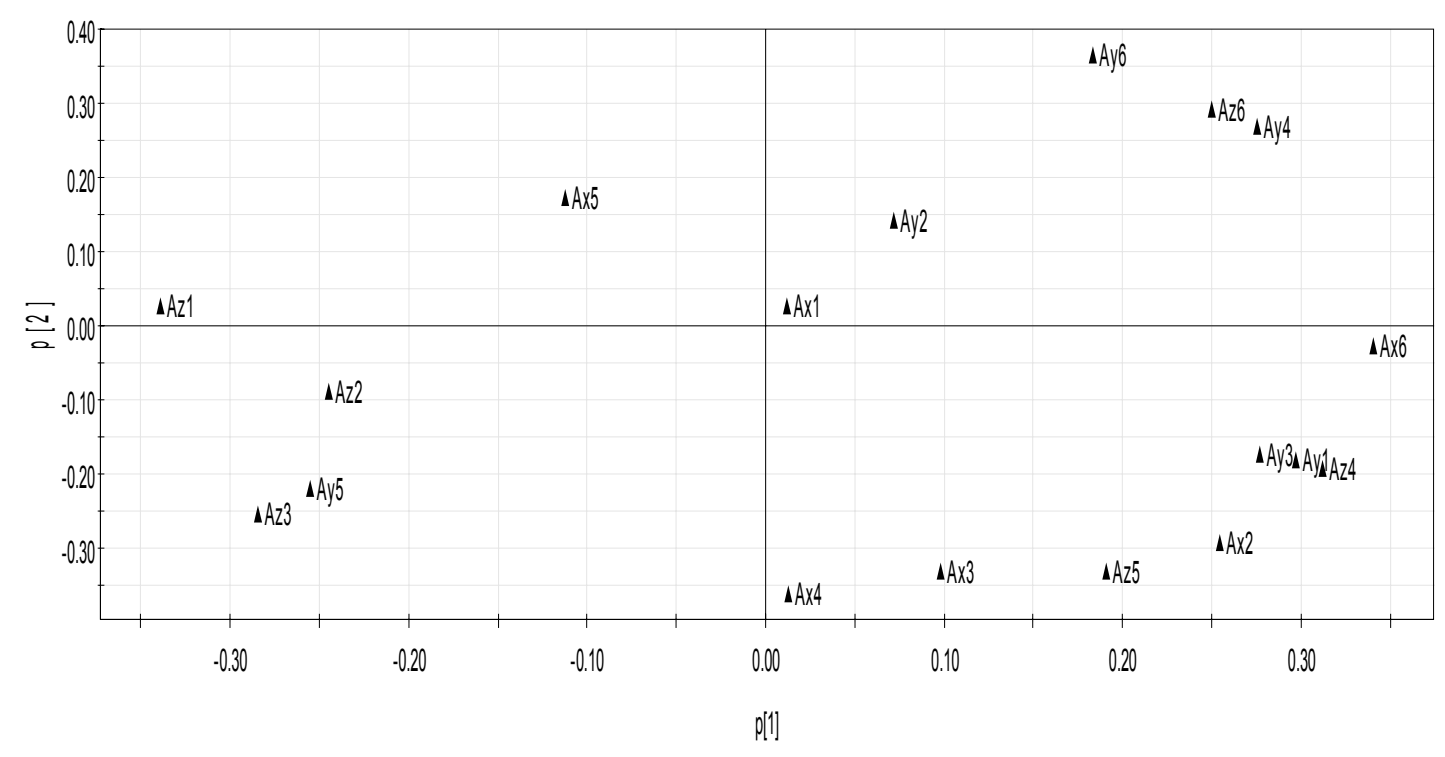

Figure 12: Loading plot of PCA model for new belt data (different speeds)



Figure 13: PCA model evaluation of the 18 parameters

A final PCA model was built on the new belt data set by cross-validation to obtain a two component model with: R2X (cum): 0.982, Q2 (cum): 0.921. Figure 14 illustrates a scatter plot of the two score vectors (t1 and t2) of the PCA model using the new belt data set. It can be seen the same three main groups representing the different machine speeds.

Figure 15 illustrates the model residual DModX where most of the observations lies under the critical distance. This means the capability of using the model for monitoring upcoming observations. 




Figure 14: Score plot of PCA model for new belt data (different speeds)



Figure 15: DModX of PCA model (residual)

Finally, control charts must be established to monitor the whole process. Figure 16 shows the Hotelling's $\mathrm{T}^{2}$ and DModX (model residual) plots with the whole data set (data with new and worn belt). It is noticed that although the model $\mathrm{T}^{2}$ didn't give any alarm in the case of a worn belt data, but the model residual DModX detected an abnormal signal starting from obs. 3073, which represents the start point of a new event or a shift in the process. Hence, In case of the occurrence of wear (not modeled) or any new events, the PCA model is alarmed with an occurrence of this new event through the model residual DModX. Hence, the model output is not used in this case and the contribution plots are checked to assign the major variables responsible for this shift.

\section{CONCLUSIONS AND FUTURE WORK}

Multivariate projection techniques are statistical methods, which can be used to describe, analyze and model multivariate data, while simultaneously measuring several 

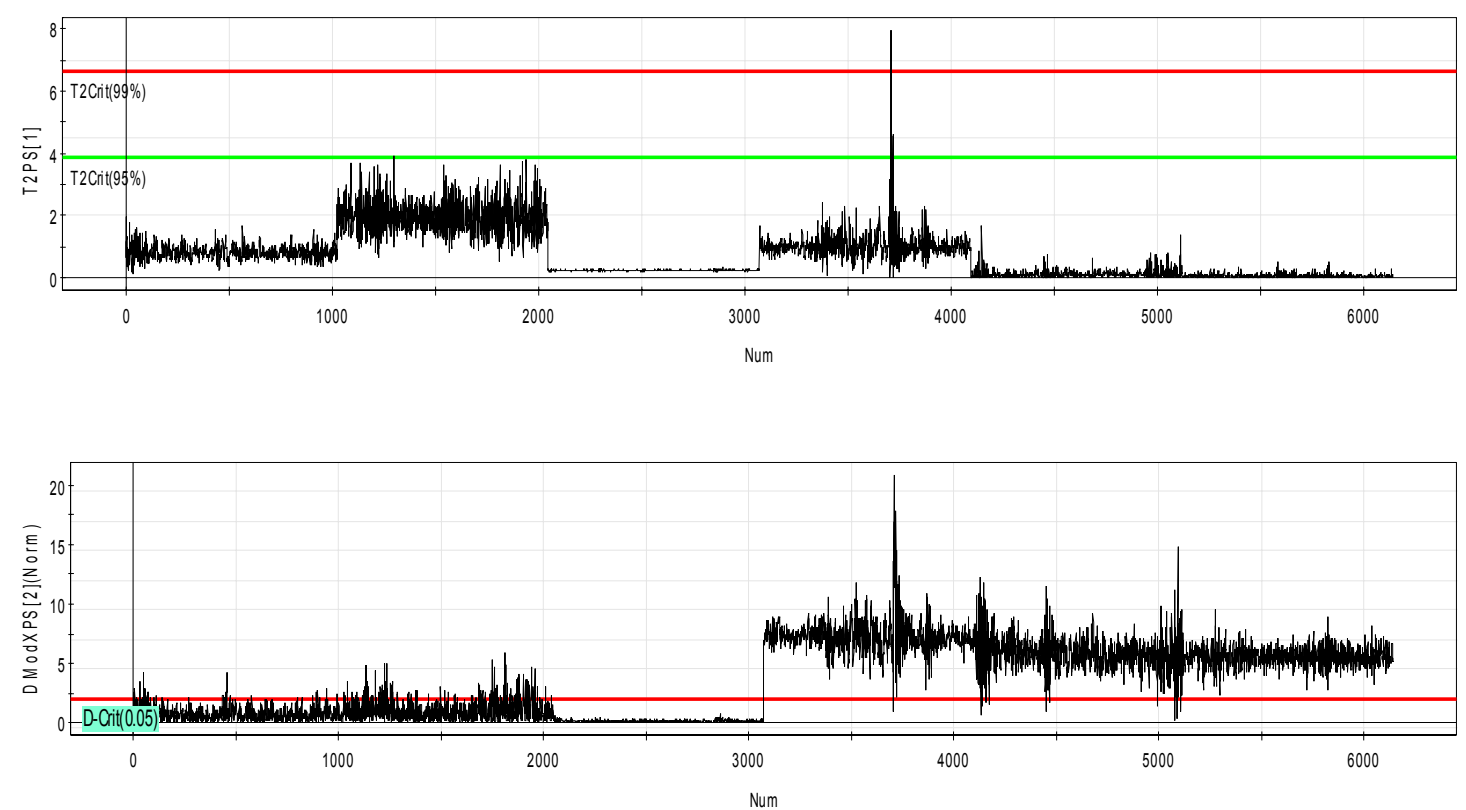

Figure 16: Shewart control chart (Training and Test sets)

predictors and response variables. The application of multivariate techniques to monitor a printing machine is the main contribution to the development of a generic machine monitoring, fault detection and optimization system. The approach uses experimental data for model building and testing. Multivariate approaches model the data in the Xspace where the advantage of the contribution plots can be seen for machine monitoring. These plots facilitate the detection of the variables responsible for any condition change both those modeled or unmodeled. Through the use of a well selected system of sensors which augments the matrix of input variables (X-matrix) with key feature components from these signals, a machine components condition can be detected.

The following recommendations are given to cover other aspects that should be tested before the application of the approach: the system has to be connected to the control system to complete the loop and achieve an automated monitoring system. More faults have to be tested from the machine itself like spindle bearing, gears and other practical issues. This can be tested by getting the geometrical dimensions of the parts and by supplying the model with their frequency components to test the classification and fault detection ability of the model.

\section{REFERENCES}

[1] J. J. Ding, "A Linear Regression Model for the Identification of Unbalance Changes in Rotating Machines", Journal of Sound and vibration, 231(1), pp.125144, 2000.

[2] Zeki Kıral and Hira Karagulle, "Vibration analysis of rolling element bearings with various defects under the action of an unbalanced force", J. Mechanical Systems and Signal Processing 20, pp. 1967-1991, 2006. 
[3] Wilson q. Wang, Fathy Ismail and M. Farid Golnaraghi, "Assessment of Gear Damage Monitoring Techniques Using Vibration Measurements", J. Mechanical Systems and Signal Processing 15(5), pp. 905-922, 2001.

[4] Yimin Zhan and Viliam Makis, "A robust diagnostic model for gearboxes subject to vibration monitoring", Journal of Sound and Vibration 290, pp. 928-955, 2006.

[5] MacGregor J.F., Yu H., Munoz S. and Cerrillo J., "Data-based latent variable methods for process analysis, monitoring and control", Journal of Computers and Chemical Engineering 29, 1217-1223, 2005.

[6] P. Nomikos and J. MacGregor, "Monitoring batch processes using multiway principal component analysis", AIChE J., Vol.40, 1361, 1994.

[7] Eriksson L., Johansson E., Wold N.K. and Wold S., "Multi- and Megavariate data analysis, principles and applications"; Umetrics AB, Sweden, 2006.

[8] Kourti T. and MacGregor J. F., "Multivariate SPC methods for process and product monitoring", Journal of quality technology, Vol.28, 4, October, 1996.

[9] Wold S., Esbensen K. and Geladi P., "Principal component analysis", Chemometrics and intelligent laboratory systems, 2: 37-52, 1987.

[10] Jackson J.E., "A User's Guide to Principal Components", Wiley, New York, 1991.

[11] Wold S., Sjostrom M. and Eriksson L., "PLS-regression: a basic tool of chemometrics", Chemometric and intelligent laboratory systems, 58:109-130, 2001.

[12] Lennox B., Hiden H.G., Montague G.A., Kornfeld G. and Goulding P.R., "Application of multivariate statistical process control to batch operation", J. Computers and chemical engineering, V24, n2, July, PP. 291-296, 2000.

[13] Goulding P.R., Lennox B., Sandoz D.J., Smith K.J. and Marjanovic, "Fault detection in continuous processes using multivariate statistical methods", International journal of systems science, 31(11), pp. 1459-1471, 2000.

[14] Yon S., Landry J., Wold N.K., Pepe and Wold S., "Multivariate process monitoring and early fault detection (MSPC) using PCA and PLS", Plant automation and decision support conference, September 21-24, San Antonio, Texas, 2003.

[15] Chen J. and Liu K.C., "On-line batch process monitoring using dynamic PCA and dynamic PLS models", J. Chemical engineering science 57:63-75, 2002.

[16] Kourti T., "Abnormal situation detection, three-way data and projection methods robust data archiving and modeling for industrial applications", Annual reviews in control 27: 131-139, 2003.

[17] P. Nomikos and J. MacGregor, "Monitoring batch processes using multiway principal component analysis", AIChE J., Vol.40, 1361, 1994.

[18] MacGregor, J.F.;" Using on-line process data to improve quality"; 1995, ASQC Statistics Division Newsletter, Vol. 16, No.2 